\title{
First beam loss measurements in the SuperKEKB positron ring using the fast luminosity monitor diamond sensors
}

\author{
D. El Khechen, ${ }^{1, *}$ Y. Funakoshi, ${ }^{2}$ D. Jehanno, ${ }^{1}$ V. Kubytskyi, ${ }^{1}$ Y. Ohnishi, ${ }^{2}$ \\ Y. Peinaud, ${ }^{1}$ C. Rimbault, ${ }^{1}$ S. Uehara, ${ }^{2}$ and P. Bambade ${ }^{1}$ \\ ${ }^{1} L A L$, Université Paris-Sud, CNRS/IN2P3, Université Paris-Saclay, Orsay, France \\ ${ }^{2}$ High Energy Accelerator Research Organization, Tsukuba, Ibaraki, Japan
}

(Received 5 June 2018; published 19 June 2019)

\begin{abstract}
SuperKEKB is a very high luminosity $e^{+} e^{-}$collider consisting of a low energy ring (LER) of positrons of $4 \mathrm{GeV}$ and a high energy ring (HER) of electrons of $7 \mathrm{GeV}$. The high luminosity will be achieved thanks to the nanobeam scheme, where very strongly focused beams $\left(\sigma_{y}=60 \mathrm{~nm}\right)$ will collide at a large crossing angle of $83 \mathrm{mrad}$. To maintain high luminosity operation, fast luminosity monitoring is essential in the presence of dynamic instabilities, for feedback and optimization. Fast luminosity monitors consisting of a single crystalline diamond sensor (LAL), a Cherenkov and scintillator detector (KEK) were placed in both rings downstream of the IP (interaction point) to measure the signal from the radiative Bhabha process at zero photon scattering angle ( $\sigma \approx 200$ mbarn). During single beam commissioning of SuperKEKB, the fast luminosity monitors were used as beam loss monitors to measure the single beam losses (beam-gas bremsstrahlung, Touschek scattering, Coulomb scattering). In this paper, a detailed description of the experimental setup, the data acquisition scheme, the data analysis and the comparison with the simulation for the diamond sensors, will be reported.
\end{abstract}

DOI: $10.1103 /$ PhysRevAccelBeams.22.062801

\section{INTRODUCTION}

SuperKEKB [1], the upgrade of KEKB, is an asymmetric $e^{+} e^{-}$collider aiming at searches for new physics beyond the Standard Model through precision measurements in the $\mathrm{B}$ meson sector. It has been designed to achieve a luminosity 40 times higher than that of KEKB $\left(8 \times 10^{35} \mathrm{~cm}^{-2} \mathrm{~s}^{-1}\right)$, which will make it the highest luminosity $e^{+} e^{-}$collider that has ever existed. To be able to maintain a very high luminosity during operation in the presence of ground motion and other disturbances, a dithering feedback system is employed to control the horizontal orbit of the beams at the IP [2,3]. The dithering system consists of a set of magnets which will kick the beam at a frequency of $77 \mathrm{~Hz}$. To monitor the effect of such kicks on the alignment of the beams and thus on the luminosity, a fast luminosity monitoring is required as input to the feedback system. Several technologies have been developed and will be compared during machine operation, single crystal chemical vapor deposition diamond (sCVD) sensors, described in this paper, as well as Cherenkov and

\footnotetext{
*Now at CERN, Switzerland

Published by the American Physical Society under the terms of the Creative Commons Attribution 4.0 International license. Further distribution of this work must maintain attribution to the author(s) and the published article's title, journal citation, and DOI.
}

scintillator detectors similar to those used at KEKB [4]. We aim at a relative precision of $10^{-2}$ in $1 \mathrm{~ms}$ and we measure the signals from the Bhabha process at zero scattering angle, which has a large cross section at the IP of about 200 mbarn. The sensors are placed in both rings just outside the beam pipe, several meters downstream of the IP. For high quality measurements, the signals in our sensors should not be contaminated by background signals from single beam losses. Detailed simulations of single beam losses were performed in order to optimise the sensitivity of our devices to the Bhabha process [5]. In this paper, after briefly reviewing the SCVD sensors used and the SuperKEKB commissioning process, we describe the main single beam loss mechanisms and assess the impact on our fast luminosity monitoring. The described studies concentrate on the setup in the LER (see Table. I), which has the largest counting rates and will be the primary device for fast luminosity monitoring.

\section{SuperKEKB PHASE I COMMISSIONING}

The SuperKEKB commissioning is split in three phases and each phase has its defined goals. The Phase I commissioning was a single beam commissioning and it took place starting from February until June 2016. During this period, BEAST II, a subgroup of the Belle II collaboration, was pursuing single beam loss studies near the IP of SuperKEKB, in order to enable assessments of the background conditions [6] within the Belle II inner detector 
TABLE I. The parameters of the positron beam in the LER during phase I commissioning.

\begin{tabular}{lc}
\hline \hline Parameter & \\
\hline Beam energy (GeV) & 4 \\
Beam current (A) & 1.1 \\
no. of bunches per beam & 1576 \\
rf acceptance (\%) & 2.8 \\
Momentum acceptance (\%) & $1-1.5$ \\
Bunch length (mm) & 6 \\
Energy spread & $7.52 \times 10^{-4}$ \\
\hline \hline
\end{tabular}

volume [7,8]. Our luminosity monitors (diamonds, Cherenkov, and scintillators monitors) also took data during that period. This commissioning phase of SuperKEKB took place without the final focus system and without the Belle II detector. Both positron and electron beams were successfully produced, accelerated, injected and stored in the main rings (LER and HER) of SuperKEKB. The main goals of this phase [9] were to start each hardware system of the machine (injector linac, magnet system, rf system in both rings, vacuum system, etc..) and to establish the beam operation software based on SAD [10]. Vacuum scrubbing was performed in both rings at different currents, starting from very low currents $(30 \mathrm{~mA})$ to high currents $(1 \mathrm{~A})$, in order to clean the vacuum chambers from residual gases, which have a large impact on single beam losses. A vacuum pressure of less than $10^{-7} \mathrm{~Pa}$ was successfully achieved at high currents in both rings.

\section{OUR PROJECT GOALS}

Our project is to perform fast luminosity monitoring [11] as part of the dithering feedback system in the horizontal plane at SuperKEKB. Furthermore, in order to achieve the aimed precision, our sensors need to be placed at locations where contamination from single beam backgrounds is minimal $(<1 \%)$ [5]. Therefore, studying the single beam loss processes is important for our project as well. We aimed from this data acquisition to address several aspects. We first wanted to check the operation of our sensors and overall installation (electronics and mechanics) under machine conditions. We then wanted to measure the backgrounds at the locations of the sensors chosen for the luminosity measurements in both rings. Finally, by comparing the data to the simulation, we wanted to check the reliability of our simulations for the subsequent commissioning phases and for luminosity operation, in order to be able, by extrapolation, to estimate the ratio of the luminosity signals to backgrounds in our sensors.

\section{DIAMOND SENSORS}

Diamond sensors (DS) provide fast signals of a few ns thanks to the high mobility of the electrons and holes generated by the ionizing particles that traverse them.
This makes them suitable to separately detect the signals from the individual bunches of SuperKEKB, which at the highest luminosities will be separated by only 4 ns. Separate detection of bunch luminosities is foreseen in addition to overall luminosity monitoring, in order to assess variations along the SuperKEKB bunch train. In addition DS are very tolerant to radiation thanks to the high binding energy [12].

The DS operation is very simple. A charged particle traverses the DS creating $e^{-} / \mathrm{h}$ pairs. A high voltage is applied to the electrodes on each side of the diamond. The oppositely charged electrons and holes generated by the ionization then drift to the electrodes. The output signal resulting from the voltage drop in the readout circuit is measured by an oscilloscope. Amplification is necessary in the case of small signals, i.e., low intensity of incident particles and/or a very thin DS. One MIP (minimum ionizing particle) creates $36 e^{-} / \mathrm{h}$ pairs per $\mu \mathrm{m}$ in the diamond. The energy deposition in the DS is a Landau

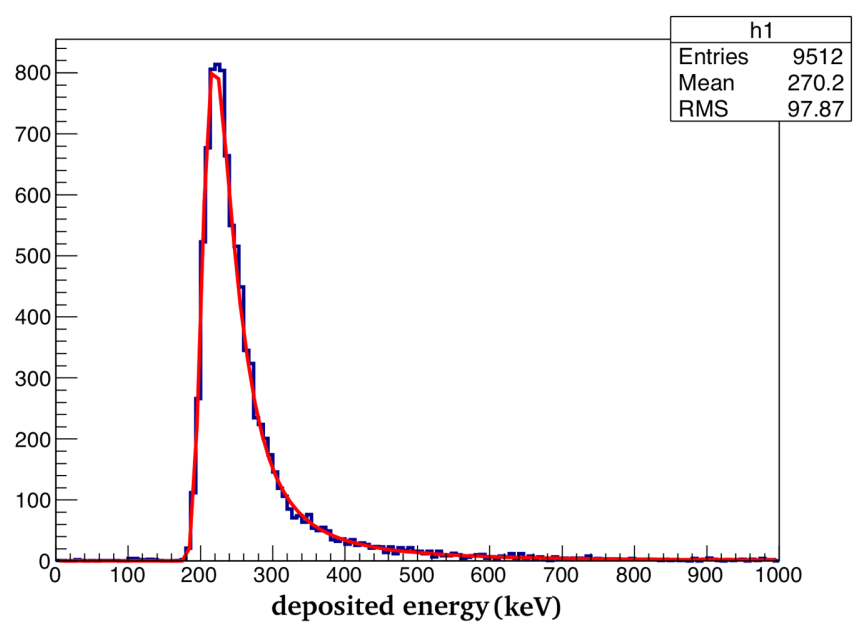

(a)

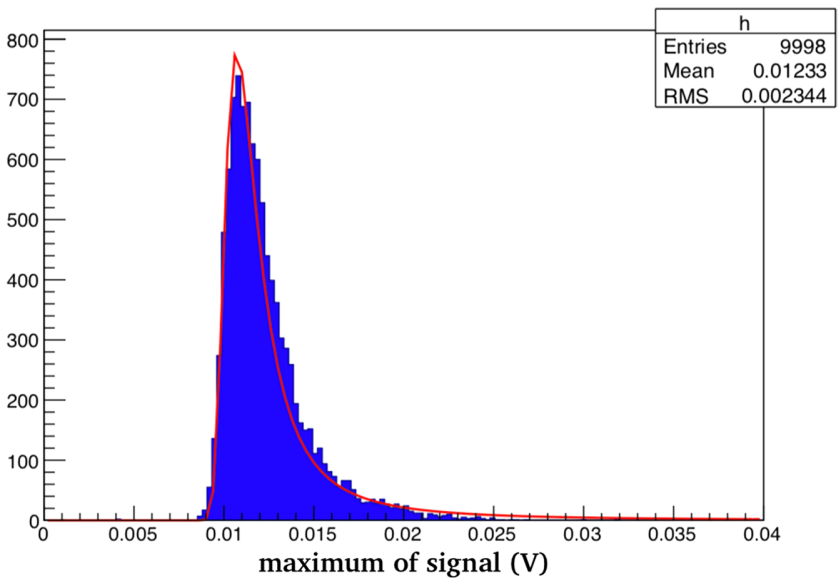

(b)

FIG. 1. (a) Simulated Landau distribution of energy deposited by MIPs in $500 \mu \mathrm{m}$ DS (b) Experimental Landau distribution in $500 \mu \mathrm{m}$ DS. 
distribution, as confirmed by performing a GEANT4 simulation using a $500 \mu \mathrm{m}$ DS with a beam of incident electrons of $3 \mathrm{GeV}$ [Fig. 1(a)] and as observed from measurements done using a ${ }^{90} \mathrm{Sr}$ radioactive $\beta$ source [Fig. 1(b)].

For all the measurements performed during the initial commissioning of SuperKEKB and described here, we used $500 \mu \mathrm{m}$ DS connected to charge amplifiers with a gain of $4 \mathrm{mV} / \mathrm{fC}$ and shaping time constant close to $10 \mathrm{~ns}$, both supplied by CIVIDEC [13].

\section{SIMULATIONS OF SINGLE BEAM LOSSES}

Beam-gas bremsstrahlung, Touschek and Coulomb scattering processes can generate single beam losses throughout the ring from the interaction of the beam particles on residual gas molecules or among themselves. In addition to being lost inside the interaction region (IR) and potentially inducing backgrounds in the Belle II detector, particles from single beam losses are lost all over the ring which reduces the beam intensities after a certain time. In rings such as SuperKEKB and due to the very high currents and the very small beam sizes, beams are expected to have relatively short lifetimes of the order of 10 minutes for the highest luminosity operation, which will require the beams to be continuously reinjected to maintain their intensity over time.

The simulation was performed using a version of the SAD code, developed at KEK to simulate single beam losses throughout the rings at SuperKEKB and to calculate the corresponding loss rates [1]. The mentioned SAD code consists of two main parts, one which generates the single beam backgrounds, tracks scattered charged particles and produces files with the tracking results, and the other which analyzes the tracking files and calculates the loss rates. Each main part calls a group of libraries responsible for different tasks (definition of the physical apertures, definition of the physics of the backgrounds, e.g., cross sections or cuts in the phase space, definition of the parameters for the tracking, especially for the scattering and observations points, definition of the calculation process of the loss rates, ...). The number of macroparticles generated at each scattering point was 2000 and the resulting particles were tracked for 30 turns in the full SuperKEKB lattice. The vertical emittance in the lattice was produced by only misaligning vertically all the sextupoles in the ring. Loss rates were calculated using appropriate weights taking into account the cross section and the parameters of the circulating beams, separately for each of the three different single beam loss processes (bremsstrahlung, Touschek and Compton), at specified observation points, either all along the ring for global loss studies, or locally at our diamond sensor. This enabled us to obtain statistically significant results, including for the dynamics (energies, positions in the vacuum chamber, angular distributions, etc...) of the tracked particles corresponding to each process, at the beginning of the drift where we placed

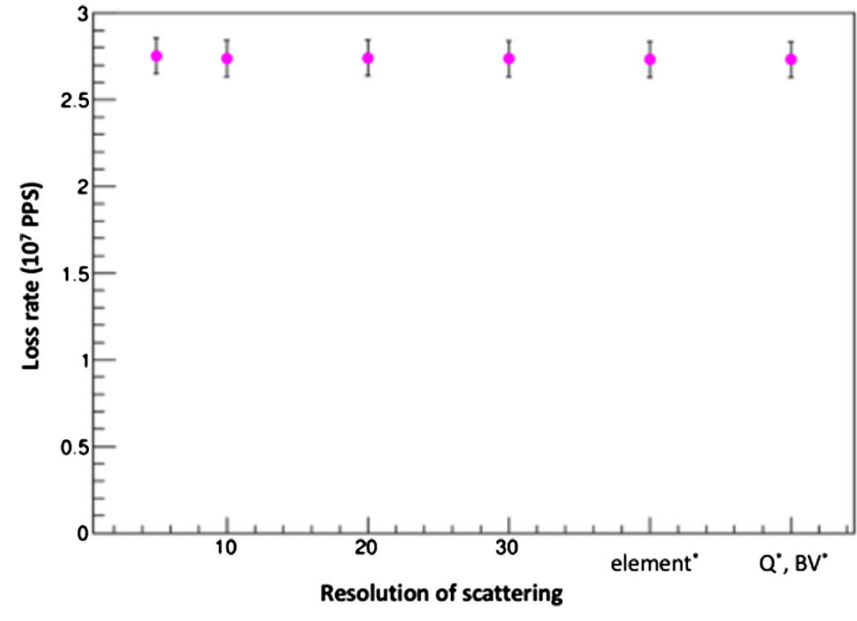

FIG. 2. The total loss from beam-gas bremsstrahlung in the LER given in particles per second (PPS) as a function of the scattering resolution in $\mathrm{cm}$ (the labels element*, $\mathrm{Q}^{*}$ and $\mathrm{BV}^{*}$ correspond to scattering only at the beginning of each element, quadrupole, or bending magnet, respectively).

our diamond sensor, to be used later to study the signal in the DS using GEANT4. The original code was further optimized to calculate the loss rates at the position of our DS with sufficient resolution and precision. To improve the precision on the simulated loss rates, the resolution of scattering positions was improved by slicing long drift spaces and bending magnets into thin slices of $10 \mathrm{~cm}$ and $15 \mathrm{~cm}$ respectively, from around 50 meters upstream of the IP to 13 meters downstream of the IP. Scattering was considered at the entrance of every element. The slicing does not affect the total loss rate in the ring (see Fig. 2) but the local loss rate at the diamond sensor position was found to increase by about $50 \%$ compared to the initial resolution where scattering was performed just at the entrance of bending magnets and quadrupoles (see Fig. 3). The drift where we installed our DS $(\approx 11.9$ meters downstream of the IP) was sliced into thin slices of $10 \mathrm{~cm}$ to get a better precision on the simulated signal in the DS. The signal in the DS was then simulated separately by a standalone GEANT4 code, taking into account the geometry of the beam pipe, its material and thickness $(6 \mathrm{~mm} \mathrm{Cu}$ cylindrical beam pipe, $40 \mathrm{~mm}$ radius physical aperture) and the dynamics of the lost particles (energy, angular distribution, etc..).

The GEANT 4 code simply defines a $3 \mathrm{~m}$ long Cu cylindrical beam pipe $(40 \mathrm{~mm}$ radius $=$ physical aperture $)$ and a $4 \times 4 \mathrm{~mm}^{2}, 500 \mu \mathrm{m}$ thick diamond sensor touching the drift.The code receives the primary particles tracked until the entrance of the drift of our DS from the SAD code, the received information consists of the energies, positions, and angular distributions of the primary positrons. Depending on their initial dynamics (mainly energy and exiting angle), the lost positron particles in the drift may or may not exit the $6 \mathrm{~mm} \mathrm{Cu}$ beam pipe (radiation length $X_{0}=1.436 \mathrm{~cm}$ ), and 


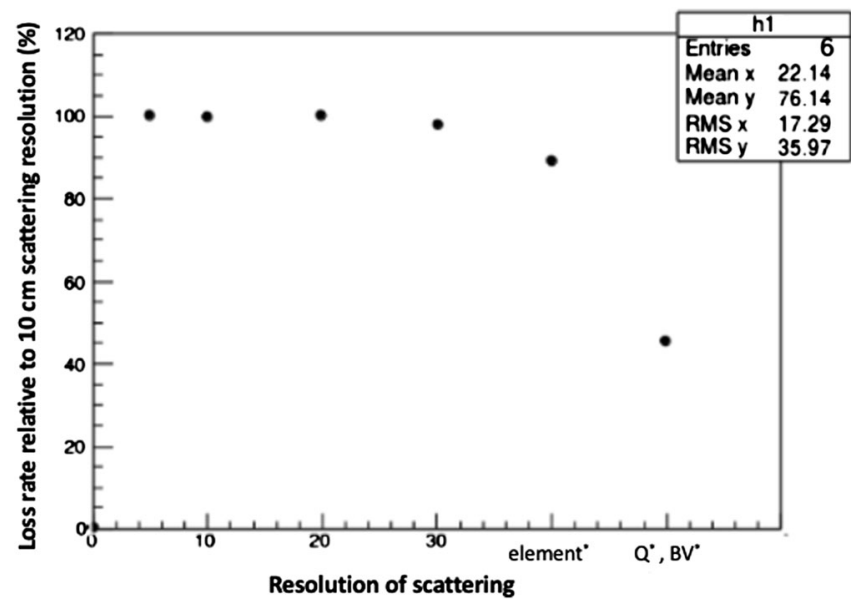

FIG. 3. The percentage of loss in the drift where we placed our DS relative to the highest resolution $(5 \mathrm{~cm})$ as a function of scattering resolution in $\mathrm{cm}$ (the labels element*, $\mathrm{Q}^{*}$ and $\mathrm{BV}^{*}$ correspond to scattering only at the beginning of each element, quadrupole, or bending magnet, respectively).

thus only a fraction of the lost positrons will deposit secondaries in the DS and create a signal. Using this code, the calculation of the signal in the DS was performed for the three different single beam loss processes.

\section{A. Beam-gas bremsstrahlung}

Beam-gas bremsstrahlung [14] is the deceleration of the charged particles of the beam when interacting with the electromagnetic field of the nucleus of the residual gas atoms in the vacuum chamber. The charged particles lose energy by emitting a photon, and can exit the beam pipe after a certain time. The energy spectrum of the scattered bremsstrahlung positrons, which are lost in the drift where we placed our DS, ranges from very low energies to the beam energy [Fig. 4(a)]. The particles lost at $11.9 \mathrm{~m}$ originate from scattering events occurring as far as about 23 meters upstream of the IP [Fig. 4(b)]. The beam-gas bremsstrahlung scattering and resulting losses are proportional to the beam current and residual pressure in the vacuum chamber (Fig. 5). Due to their rate and dynamics at the loss position and to the material and thickness of the beam pipe only a fraction of $5 \times 10^{-3}$ of the total number of lost particles will be able to give a signal in the DS.

\section{B. Touschek scattering}

Touschek scattering [15-17] is the Coulomb scattering between particles of the same bunch. Since the horizontal emittances are larger than the vertical emittances, we assume that the initial momenta of the scattering particles are in the horizontal direction. Due to the boost in the longitudinal direction, Touschek scattering can result in a maximum energy transfer between both particles given in Eq. (1), where $\gamma$ is the Lorentz factor and $p_{x}$ is the assumed initial transverse momentum of the particle. The particles

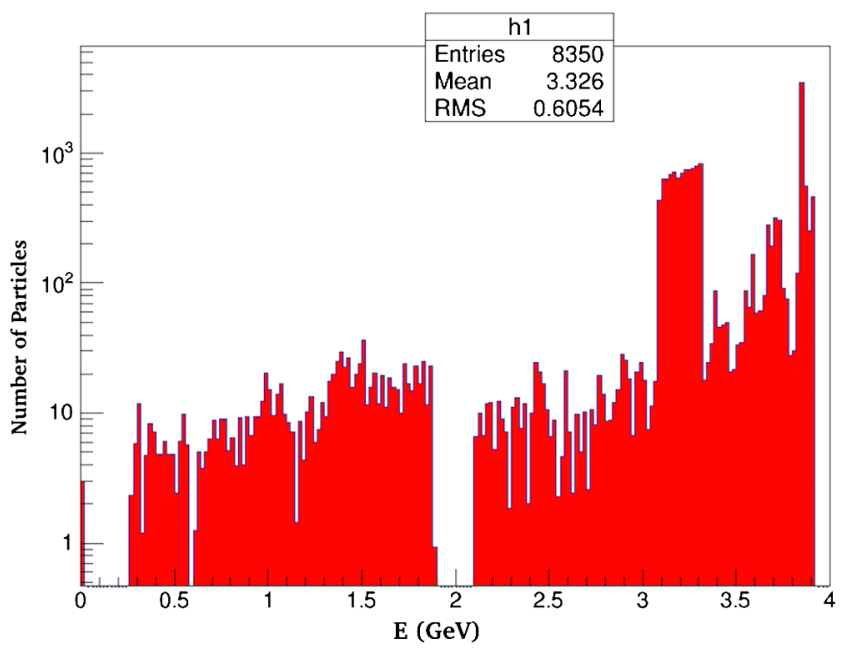

(a)

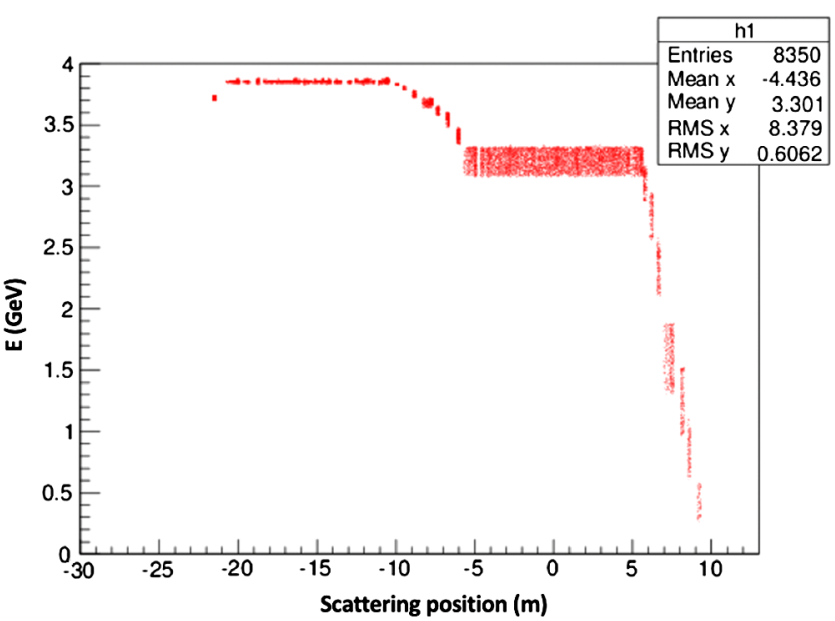

(b)

FIG. 4. (a) Simulated energy spectrum of the subset of bremsstrahlung positrons which are lost in the drift where we place our DS and (b) their energy as a function of their scattering position (0 represents the IP).

are lost if their relative energy variation $\Delta E$ exceeds the energy acceptance of the machine. The energy acceptance of SuperKEKB is about 1-1.5\%, thus if a positron gains or loses more than $0.06 \mathrm{GeV}$ or an electron gains or loses more than $0.1 \mathrm{GeV}$, they will be lost from the beam. Touschek loss rates are proportional to the square of the beam current [Fig. 6(a)] and the inverse of the beam size [Fig. 6(b)].

$$
\Delta E_{\max }=\gamma \times p_{x} \times c
$$

The Touschek scattered positrons which are lost in the drift at 11.9 meters result from scattering occurring at a maximum distance of 20 meters upstream of the IP, and they can lose or gain a maximum of $7 \%$ of their energy (Fig. 7). The particles which gained energy exit the beam pipe on the high energy side $(-x)$ with a very shallow horizontal angle, whereas the particles which lost energy 


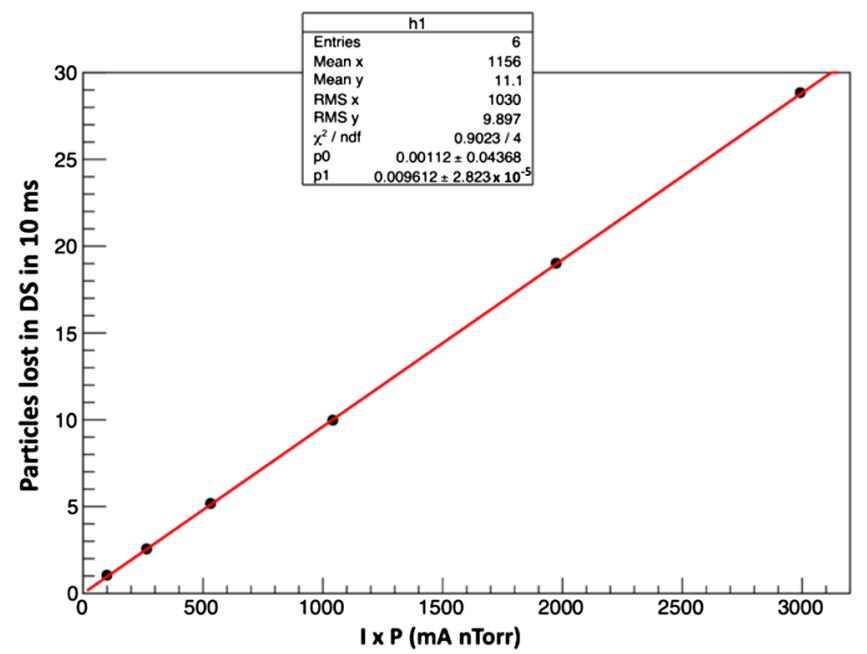

FIG. 5. Simulated behavior of the subset of bremsstrahlung losses detected in the DS as a function of the product of beam current and vacuum pressure.

exit the beam pipe on the low energy side $(+x)$ where we placed our diamond sensors.

Since the Touschek particles exit the beam pipe on both sides in the horizontal plane, they will produce charged secondaries mainly in that plane after having traversed the material of the beam pipe [Fig. 8(a)]. Each exiting charged secondary corresponds to one incident particle, nevertheless some incident particles are absorbed totally in the beam pipe with zero secondaries exiting [Fig. 8(b)]. Among all the particles that are lost in the drift only very few generate a signal in the DS (a fraction of $4.85 \times 10^{-3}$ ), by depositing secondaries. This fraction is given by the GEANT4 simulation which takes into account the exiting positions, angular distributions, and energies of the lost particles in the drift where the DS is placed. Most incident particles giving a signal in the DS deposit only one secondary, and just a few may have more than one secondary (Fig. 9).

\section{Coulomb scattering}

Coulomb scattering [18] is an elastic scattering process between the particles of the beam and the nuclei of the residual gas atoms where the particles receive a transverse kick, keeping their initial energies. The Coulomb scattered positrons at positions up to $\approx 80$ meters upstream of the IP will be lost in the drift where our DS is placed due to their transverse angles in the horizontal and vertical planes (Figs. 10a and 10b).

The Coulomb scattered positrons are lost mainly in the vertical plane (fewer particles are lost in the horizontal plane), and this can be observed clearly from the distribution of the charged secondaries which are the result of the interaction of the lost incident positrons with the material of the $6 \mathrm{~mm} \mathrm{Cu}$ cylinder beam pipe (Fig. 11a). The transverse

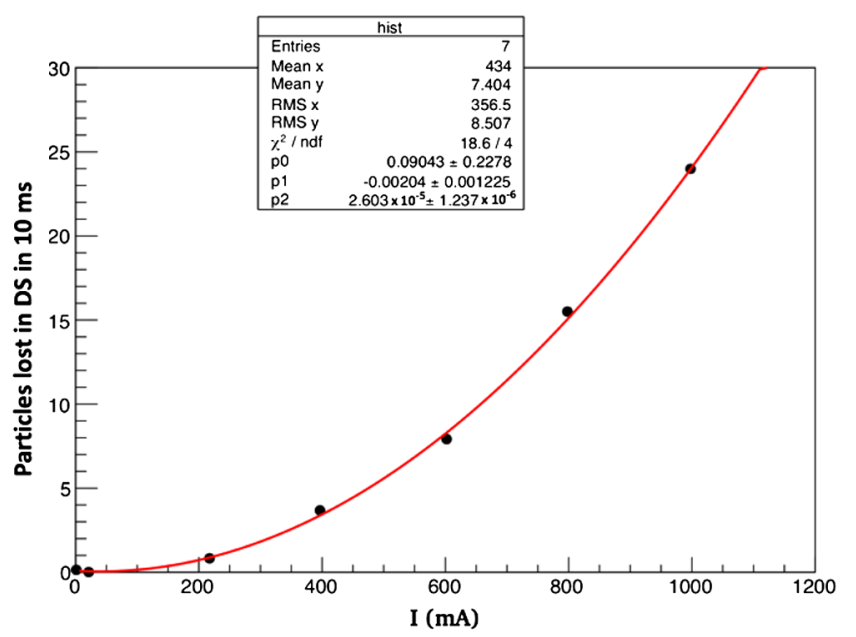

(a)

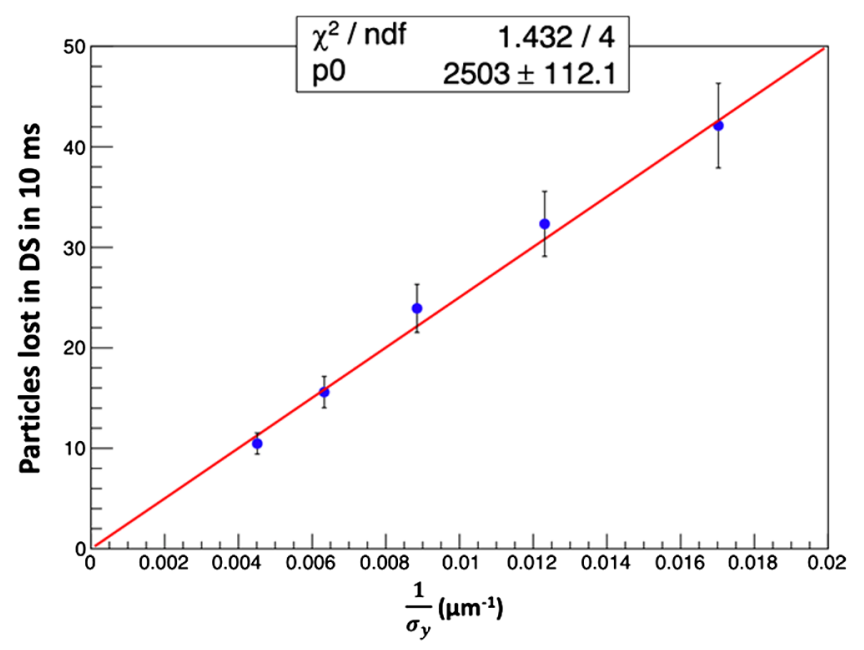

(b)

FIG. 6. (a) Simulation of expected Touschek loss rate in the DS in $10 \mathrm{~ms}$ as a function of the beam current and (b) as a function of the inverse vertical beam size.

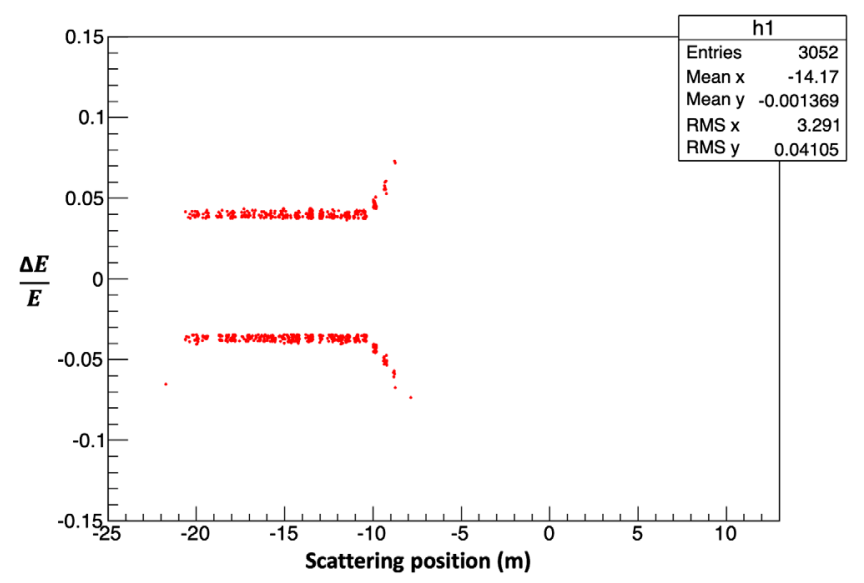

FIG. 7. The $\frac{\Delta E}{E}$ of the subset of Touschek positrons lost in the drift where we placed our DS as a function of their scattering position (from simulation). 


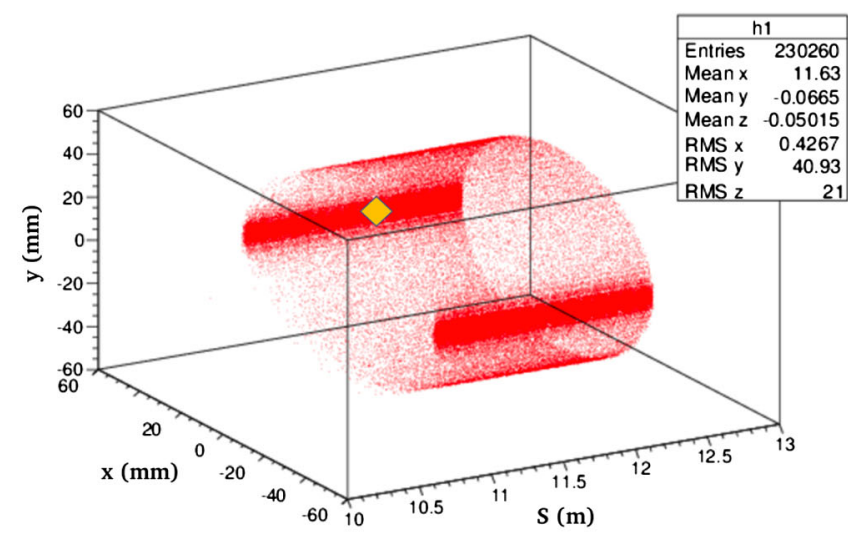

(a)

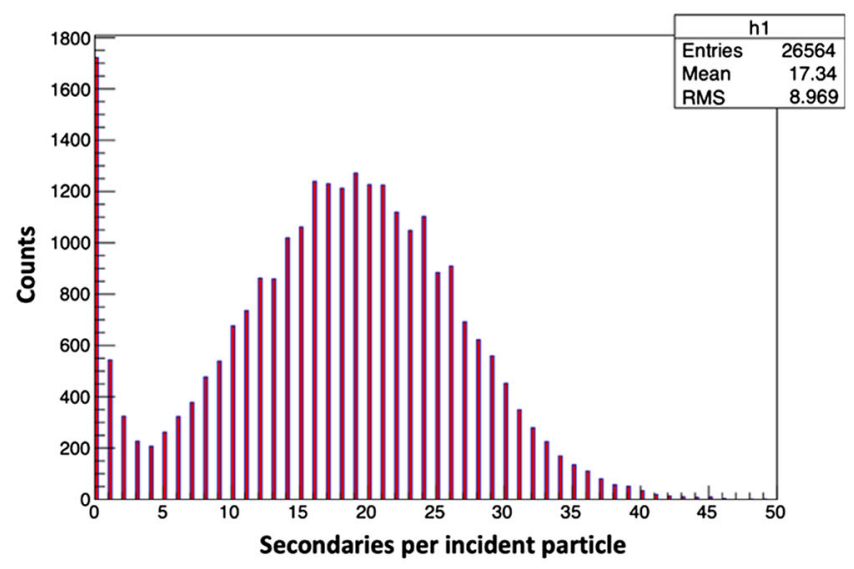

(b)

FIG. 8. (a) The 3D distribution of the charged secondaries resulting from the interaction of the subset of the lost Touschek positrons with the $6 \mathrm{~mm} \mathrm{Cu}$ cylinder beam pipe in the drift where we placed our DS (represented by a yellow diamond) and (b) their number per incident particle.

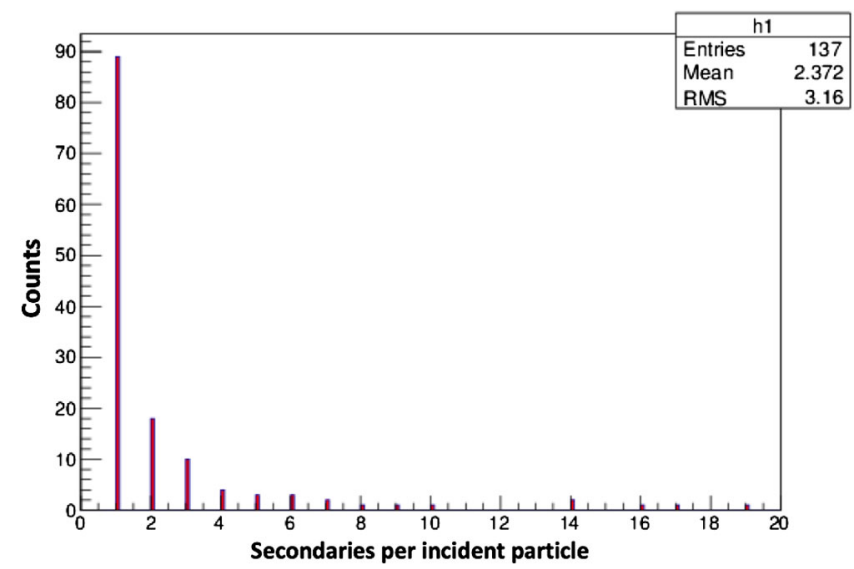

FIG. 9. Number of secondaries depositing energy in the DS by incident Touschek scattered positron.

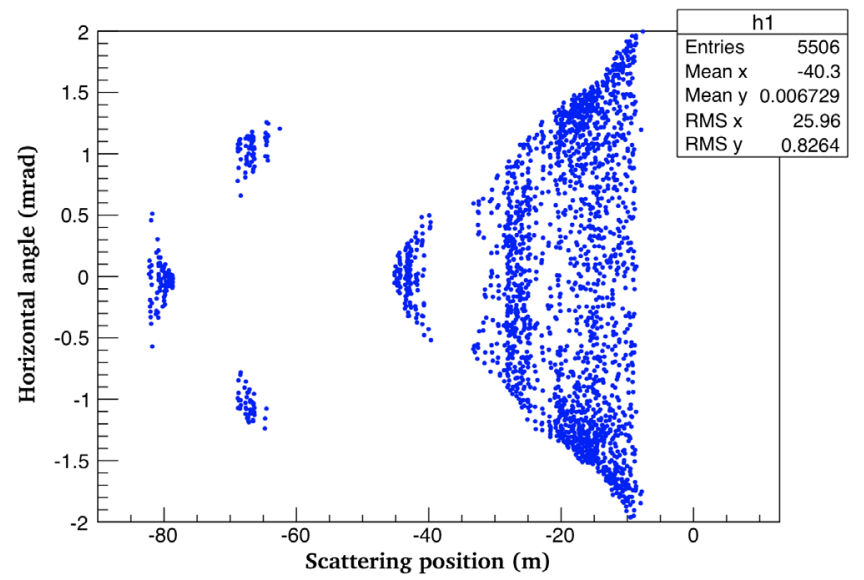

(a)

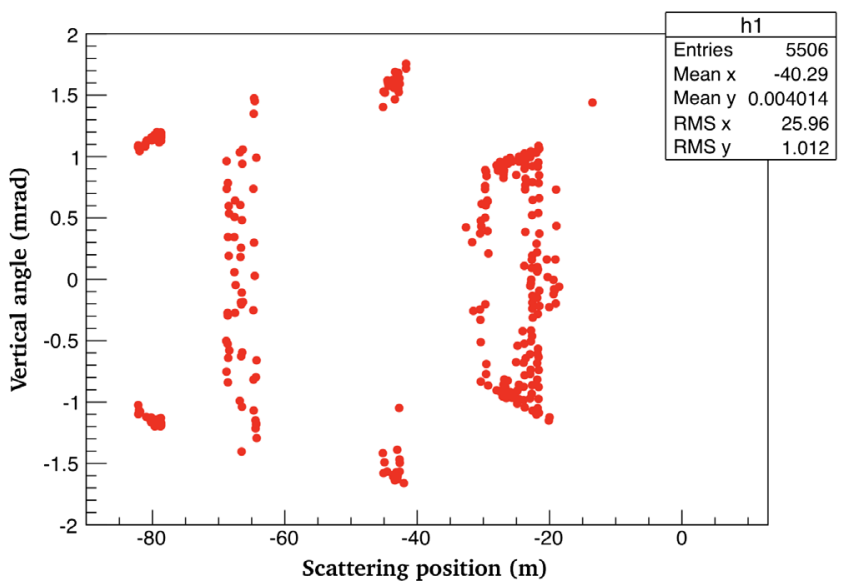

(b)

FIG. 10. (a) The horizontal exiting angles and (b) the vertical exiting angles of the lost Coulomb scattered positrons in the drift where we place our DS.

offset of a particle at a given loss position (B) due to a kick at an emission point (A) is proportional to the transfer matrix elements R12 for the horizontal plane and R34 for the vertical plane, between $\mathrm{A}$ and $\mathrm{B}$, which can be expressed as $\sqrt{\beta_{A} \beta_{B}} \sin \left(\phi_{A B}\right)$, where $\beta_{A}$ and $\beta_{B}$ are the transverse $\beta$ functions at the emission and loss positions respectively. While $\beta_{x}$ and $\beta_{y}$ are about equal at the considered loss position in the drift where the DS is located [see Fig. 11(a)], $\beta_{y}$ is larger than $\beta_{x}$ at the positions upstream in the beam line where most of the Coulomb scattering occurred [see Fig. 11(b) and 12]. This can explain why Coulomb scattered positrons are mainly lost in the vertical plane. The distribution of the scattering positions of the Coulomb positrons lost in the DS in the drift [Fig. 11(b)] shows for instance a peak at $\approx 80$ meters upstream of the IP, where the $\beta_{y}$ is larger than $\beta_{x}$. Since our DS is placed in the horizontal plane on the low energy side $(+x)$ and the Coulomb loss is mainly in the vertical plane, we basically have no signal from Coulomb scattering in the DS. 


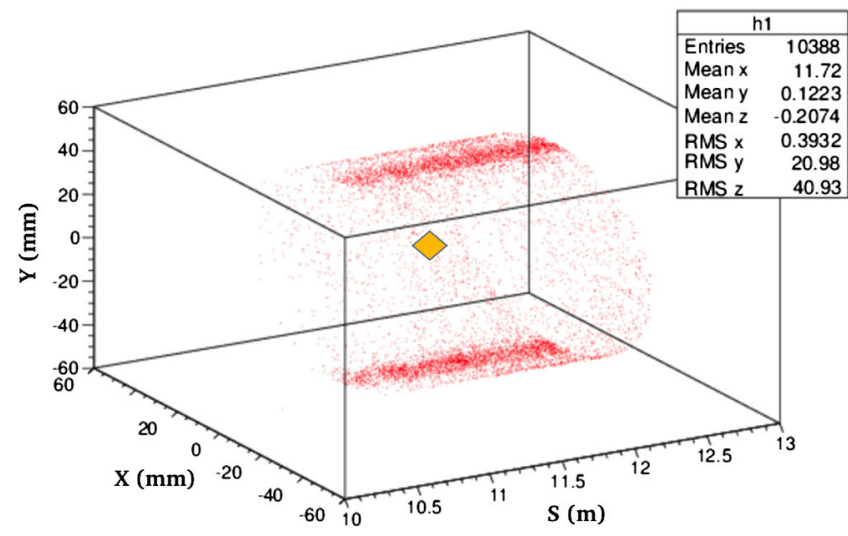

(a)

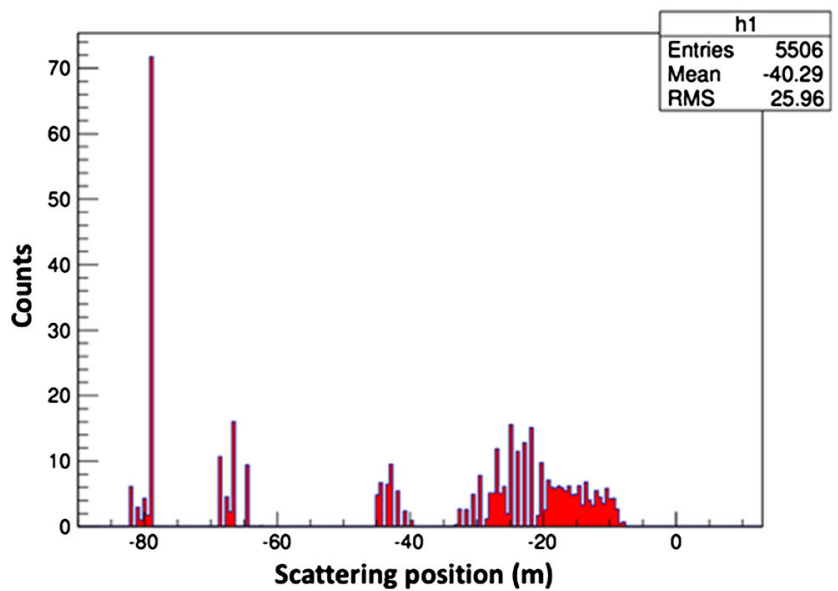

(b)

FIG. 11. (a) 3D distribution of the positions of the charged secondaries exiting the $6 \mathrm{~mm} \mathrm{Cu}$ cylinder beam pipe with the yellow diamond representing our DS (b) the scattering positions of the Coulomb positrons lost in the DS in the drift (from simulation).

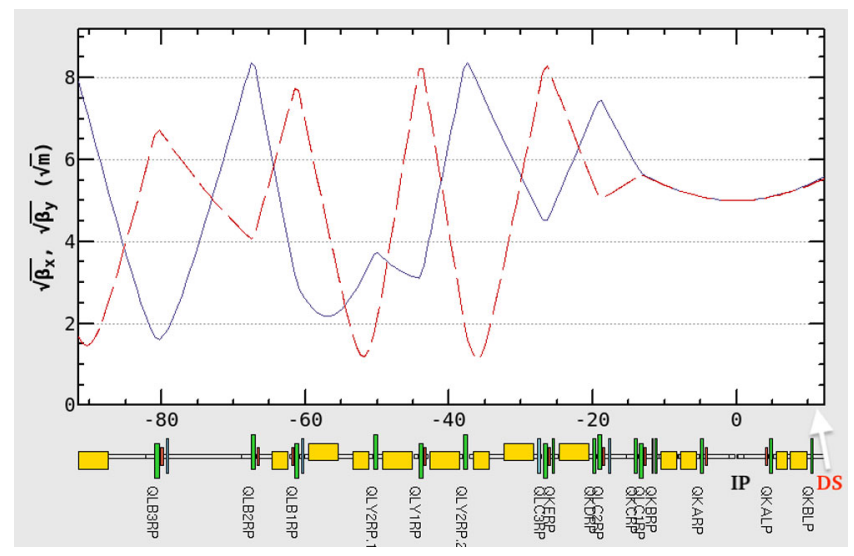

FIG. 12. The square root of the horizontal (blue) and vertical (red) $\beta$ functions at the scattering positions from $\approx 100$ meters upstream of the IP to the drift about 11 meters after the IP.

\section{MEASUREMENTS OF SINGLE BEAM LOSSES DURING PHASE I COMMISSIONING}

\section{A. Mechanical setup}

The mechanical setup shown in Fig. 13 was prepared at LAL for the LER and installed in preparation for the Phase I commissioning. A pillar located 11.9 meters downstream of the IP supports a movable plate with two sCVDs each connected to a CIVIDEC charge amplifier, and a fixed plate with the Cherenkov and scintillator counters. The movable plate is associated to a remotely controlled motor to scan along the vertical direction over a range of $2.5 \mathrm{~cm}$, and the sensors are facing a normal cylindrical beam pipe in the drift.

\section{B. Electronic readout}

For Phase I, two $\left(4 \times 4 \mathrm{~mm}^{2}, 500 \mu \mathrm{m}\right)$ sCVDs connected to CIVIDEC charge amplifiers were used in the LER. The signals from the SCVDs were sent to the electronic rack located in the Belle II experiment electronic hut by rigid, about 100 meter long, half inch heliax cables.

The electronic rack was equipped with a 10 bit Keysight oscilloscope $(2.5 \mathrm{GHz}$ bandwidth and up to $20 \mathrm{GS} / \mathrm{s}$ sampling), a PC serving as gateway to the Linux operated Belle II DAQ computers, a Windows based server on which analysis using MATLAB was performed, a high voltage power supply for the diamond sensors $(400 \mathrm{~V})$ and a low voltage power supply for the charge amplifiers (12 V).

The oscilloscope was used to record the data during Phase I commissioning, using two signal acquisition procedures, either a succession of continuous $10 \mathrm{~ms}$ records that could be processed on-the-fly every 5-6 seconds or up to fifty $410 \mathrm{~ms}$ long records spaced by few seconds, where the processing could only take place afterwards and lasted

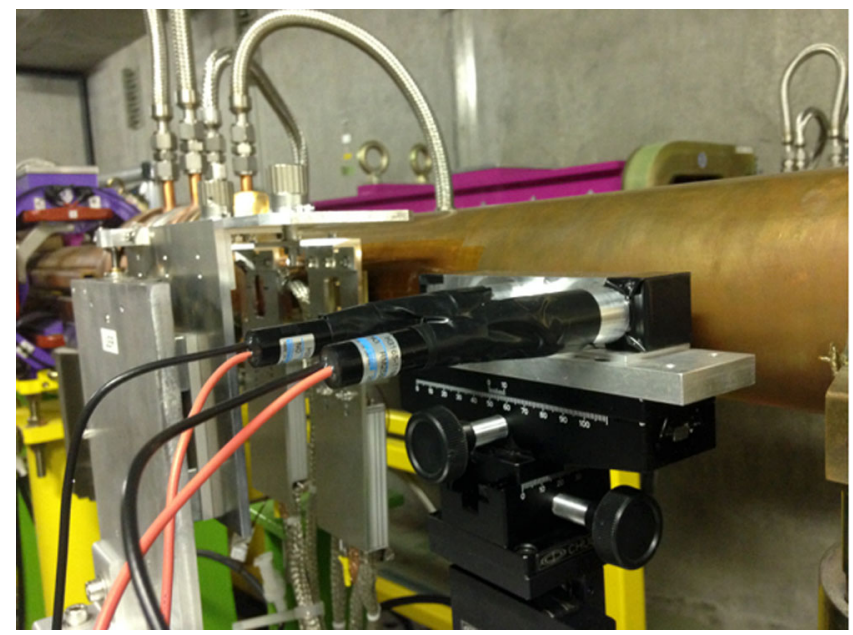

FIG. 13. The pillar in the LER located at 11.9 meters downstream of the IP holding the two sCVDs and the Cherenkov and scintillator counters. 


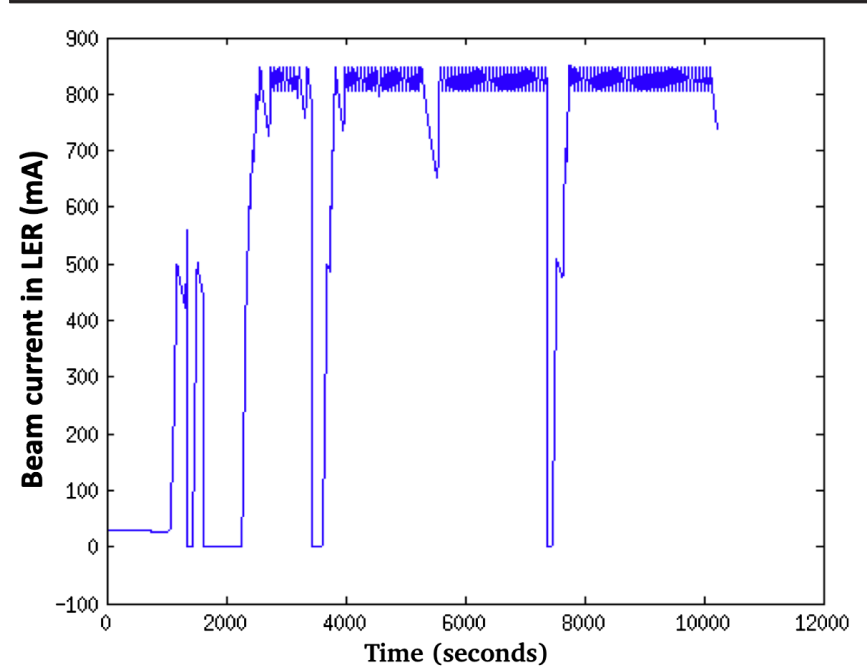

(a)

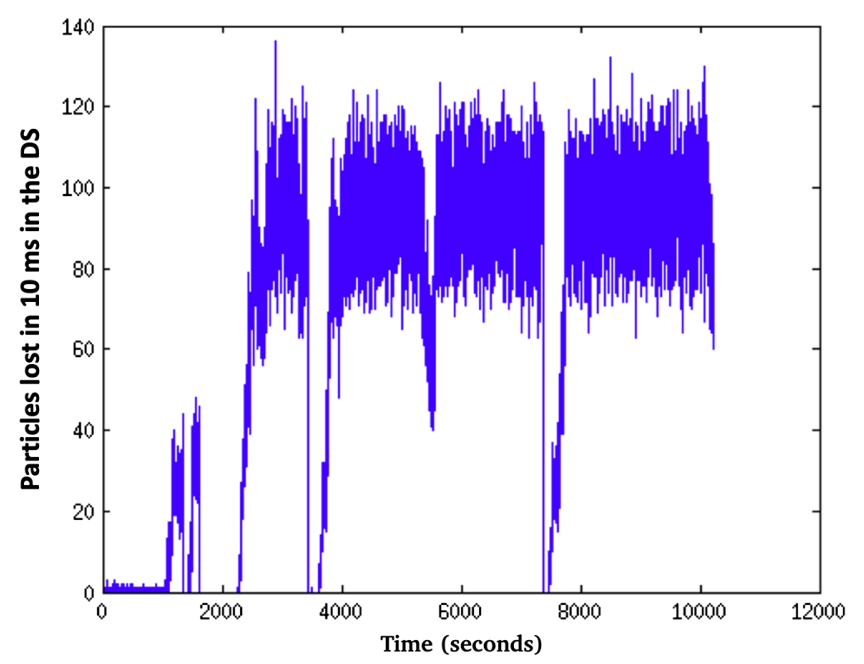

(b)

FIG. 14. (a) The beam current in the LER as function of time and (b) the loss rate measured by the sCVD in $10 \mathrm{~ms}$ over the same time period.

for a few hours. Operating with this preliminary data acquisition scheme limited the statistics and data sequences needed to be combined to reduce the errors.

Each minimum ionizing particle (MIP) generates an average charge of $2.9 \mathrm{fC}$ in the $4 \times 4 \mathrm{~mm}^{2}$ metallized part of the $500 \mu \mathrm{m}$ thick sCVD. The signal is then amplified by a charge amplifier with a gain of $4 \mathrm{mV} / \mathrm{fC}$. The MPV (most probable value) of the Landau distribution in the measurements $(11.6 \mathrm{mV})$ was observed to be 1.26 times greater than that in the GEANT4 simulations $(9.2 \mathrm{mV})$. To have a reasonable comparison between the data and the simulation, this factor 1.26 was taken into account by rescaling the threshold used in the simulation. Since acceptable separation between the noise from the charge amplifier in the measurements could be obtained by setting the threshold at $6 \mathrm{mV}$, therefore the threshold for the simulation was set to $4.75 \mathrm{mV}$.

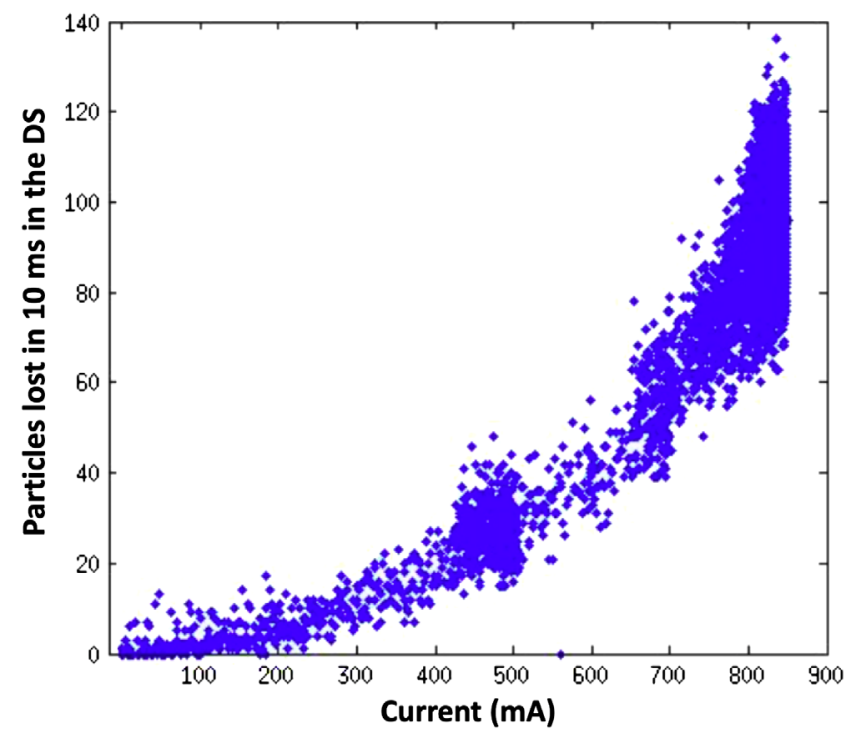

(a)

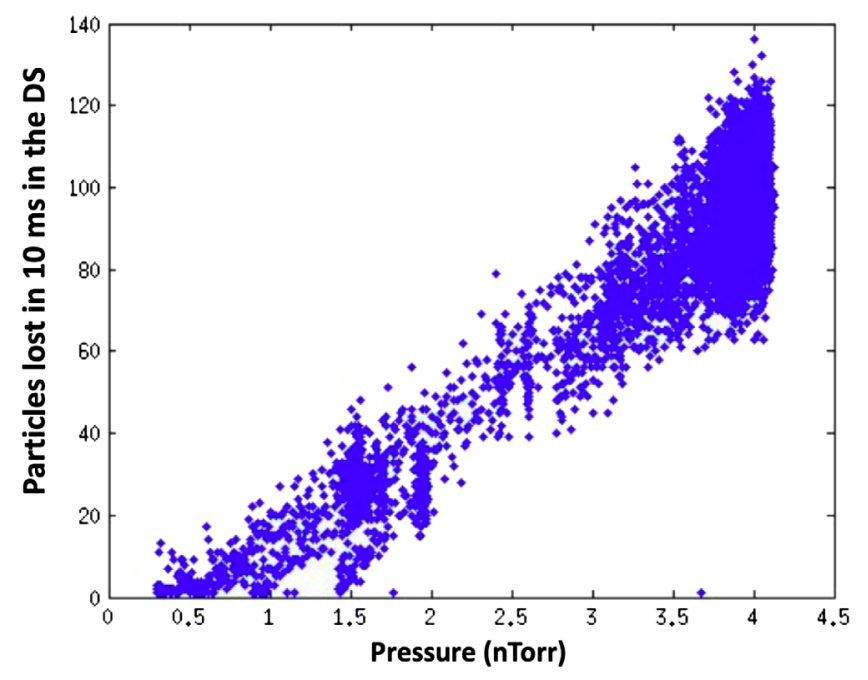

(b)

FIG. 15. (a) The measured loss rate in $10 \mathrm{~ms}$ in the SCVD in LER as a function of beam current and (b) as a function of vacuum pressure.

The first step of the data analysis consisted in finding the number of peaks in a set of acquisition sequences of $10 \mathrm{~ms}$, taking into account the threshold at $6 \mathrm{mV}$. The rates of beam loss could then be studied as a function of different beam parameters (current, vacuum pressure, vertical beam size). The analyzed data were observed to follow the beam current variations from the very first measurements, during the injection and the storage of the beam (Fig. 14). The variation of the total loss rate in the SCVD per $10 \mathrm{~ms}$ given as a function of the beam current [Fig. 15(a)] shows the expected quadratic behavior, which results in particular from the contribution of Touschek losses, and the linear variation as a function of the vacuum pressure [Fig. 15(b)], which is expected from the contribution of beam-gas bremsstrahung. 


\section{Beam loss measurements}

Several dedicated background studies were considered during the Phase I run of SuperKEKB. The aim was to understand the origin and the behavior of such losses with respect to different machine parameters. Collimation studies also took place in parallel. Once a global understanding of these backgrounds is achieved, extrapolation to the upcoming phases is needed in order to make sure the Belle II detector and the final focus system are well protected.

In the following, we describe the background study during which we were able to extract information on the contribution of each of the single beam losses, Touschek and beam-gas bremsstrahlung, in our DS.

\section{Vertical beam size scan}

The aim of the so-called "Touschek study", which took place during the Phase I commissioning was to extract the contribution of the Touschek losses from the total losses by analyzing their dependance on the vertical beam size $\sigma_{y}$. The beam size was increased by varying the value of the ECK (Emittance Control Knob), which consists in changing the current in a specific winding of the chromaticity correcting sextupoles in the LER, to generate a skew quadrupole component, thereby inducing a coupling of the horizontal emittance into the vertical plane in the ring, and thus increasing the vertical positron beam size.

During the study, beam loss data were collected at six different beam currents in the LER, $I=180,360,540,720$, 900, and $1000 \mathrm{~mA}$ (Fig. 16(a)). For each value of the current, $\sigma_{y}$ was varied five times [Fig. 16(b)], with data taking lasting for $\approx 2$ minutes at each value of $\sigma_{y}$.

Losses observed in the sCVD result from both beam-gas bremsstrahlung and Touschek processes, and there is no contribution from Coulomb scattering in the DS for the reasons mentioned in Sec. VC. For fixed values of the current (which also maintains more or less a constant vacuum pressure) and varying the vertical beam size, the contribution of the beam-gas bremsstrahlung can be expected to remain constant while that of Touschek scattering should vary. Fitting the losses in the sCVD as a function of the inverse of the vertical beam size $\left(\sigma_{y}^{-1}\right)$ allows separating both contributions in the following way. For very large $\sigma_{y}$, the losses from Touschek scattering become negligible and thus the losses in the sCVD are dominated by beam-gas bremsstrahlung. The contributions from bremsstrahlung at each beam current are thus extracted from the y-intercepts of the fits shown in Fig. 17.

\section{Losses from beam-gas bremsstrahlung}

The losses from bremsstrahlung extracted from the fits (Table II) are compared to the simulation as a function of the product of the beam current and the vacuum pressure in the LER, see Fig. 18. The fit of the losses from bremsstrahlung is linear in both measurement and simulation, but

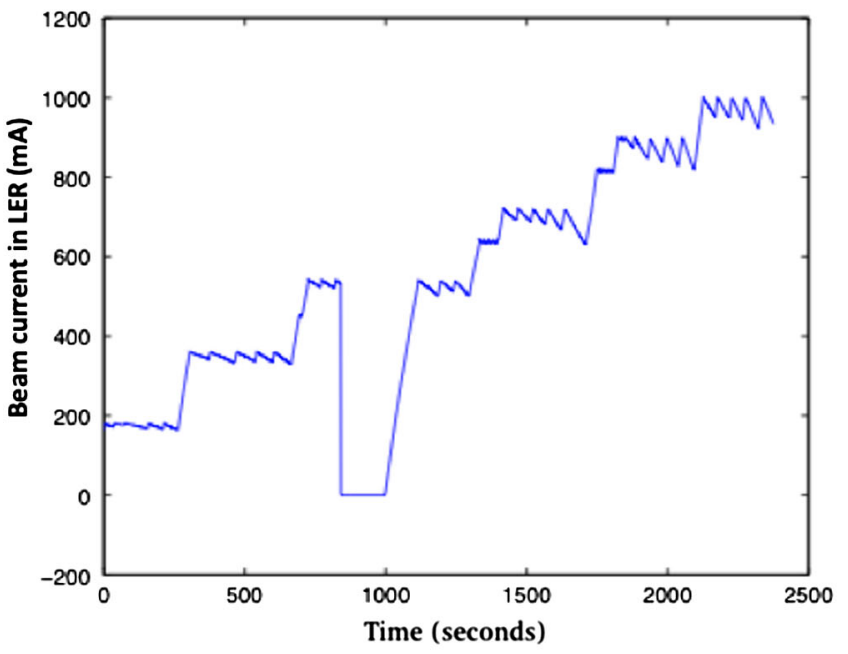

(a)

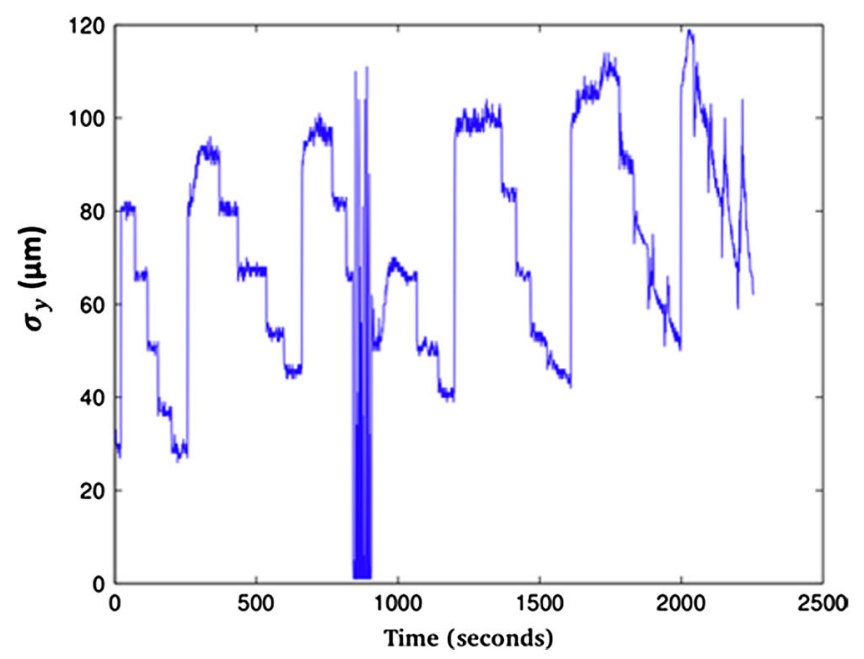

(b)

FIG. 16. (a) Beam current and (b) vertical beam size in the LER during the Touschek study.

we can see that there is a factor between the two slopes with that for the measurement always greater than that for the simulation, by about a factor of 3 (Fig. 18). This factor can be explained by the fact that the measured value of the pressure from the CCG (cold cathode gauge) underestimates the real pressure seen by the beam in the center of the vacuum chamber. In SuperKEKB, the CCGs are positioned on the NEG (nonevaporable getter) pumps, thus the measured pressure at the pumping position is lower than that in the center of the vacuum chamber. This ratio between real and measured pressures was studied in KEKB [19] where factors in the range of 1.5 to 3 were found, reasonably consistent with our observation. A more detailed modelling of the gas pressure was not pursued for SuperKEKB during the Phase I commissioning, since the vacuum chamber in the interaction region was only a temporary one, to be replaced by a narrower one for the subsequent operations with Belle II detector. 


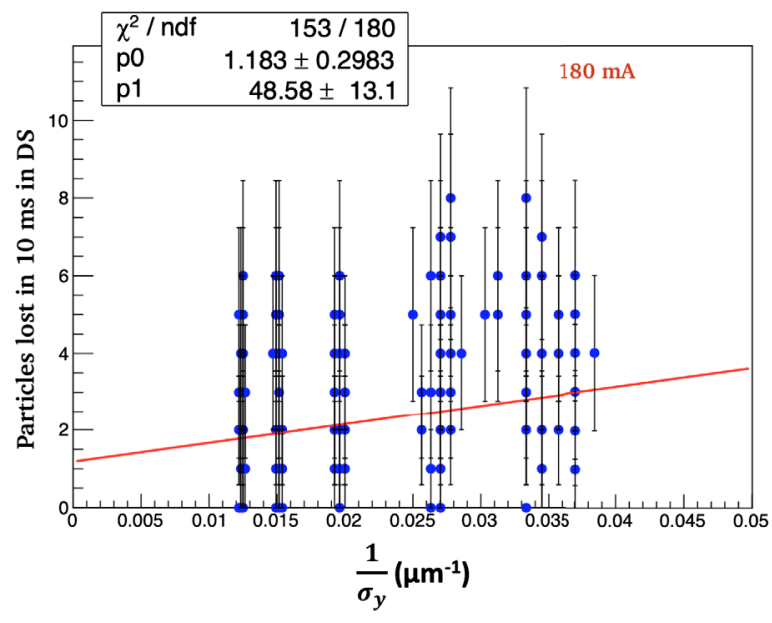

(a)

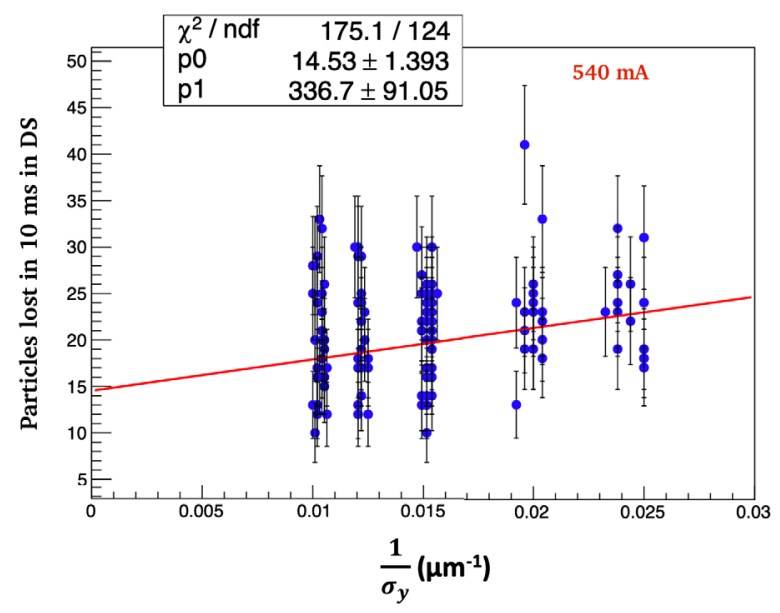

(c)

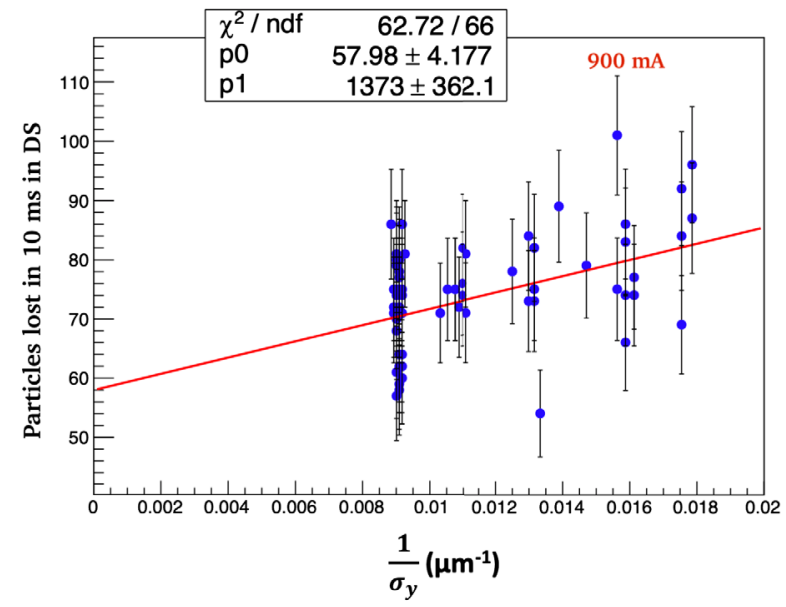

(e)

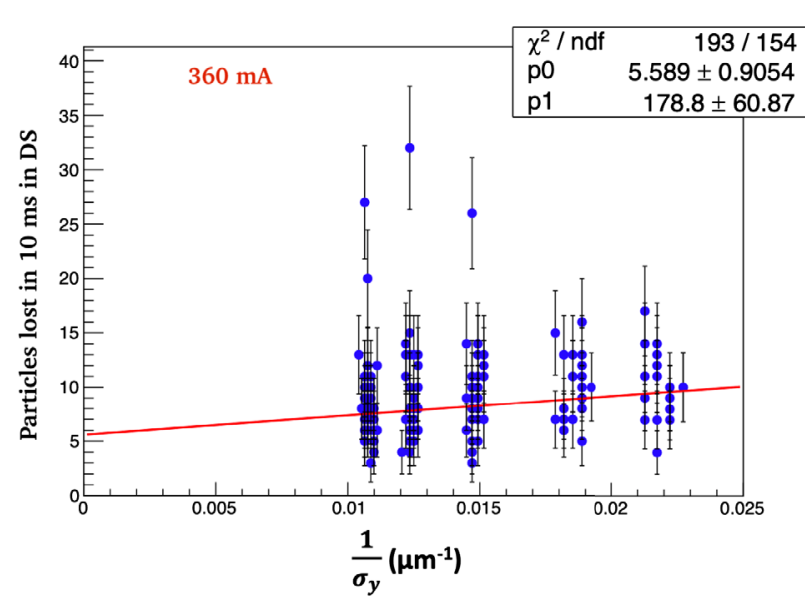

(b)

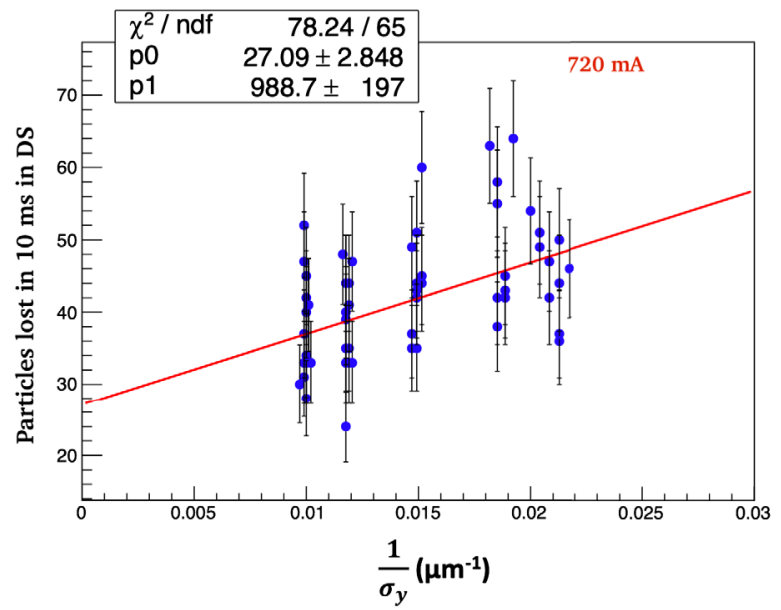

(d)

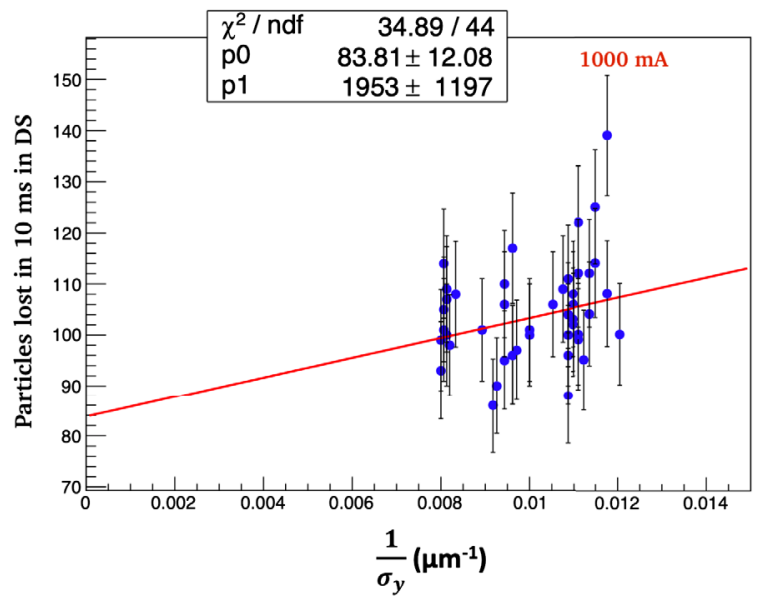

(f)

FIG. 17. Fit of the measured losses in the SCVD as a function of the inverse of the vertical beam size at different beam currents marked in red inside each plot. 
TABLE II. Measured losses from beam-gas bremsstrahlung in $10 \mathrm{~ms}$ in the DS for different beam currents and vacuum pressures in the LER.

\begin{tabular}{lcc}
\hline \hline Current $(\mathrm{mA})$ & $\begin{array}{c}\text { Pressure } \\
\text { (nTorr) }\end{array}$ & $\begin{array}{c}\text { Beam-gas } \\
\text { bremsstrahlung } \\
\text { in DS in 10 ms }\end{array}$ \\
\hline 180 & 0.56 & $1.2 \pm 0.3$ \\
360 & 0.75 & $5.6 \pm 0.9$ \\
540 & 1 & $14.5 \pm 1.4$ \\
720 & 1.45 & $27.1 \pm 2.8$ \\
900 & 2.2 & $58.0 \pm 4.2$ \\
1000 & 3 & $83.8 \pm 12.1$ \\
\hline \hline
\end{tabular}

\section{Losses from Touschek scattering}

The information on the Touschek contribution to the total beam losses in the SCVD is contained in the slopes of the fits in Fig. 17. To compare the contribution to the losses from Touschek scattering in the sCVD in measurements and simulations, the slopes of the fits in Fig. 17 were extrapolated quadratically to the same current (180 mA). Given that the bunch length calculated by the lattice is evaluated in SAD in the hypothesis of very low beam intensities and without taking into account collective effects, the slopes were also extrapolated to the bunch length in the simulation $(4 \mathrm{~mm}$, rather than about $6 \mathrm{~mm}$ at the different beam intensities in the LER). The weighted arithmetic mean of the extrapolated slopes was then computed along with the errors. The result of this calculation is Touschek $_{\text {meas }}=(70.6 \pm 8.1) \times \frac{1}{\sigma_{y}}$.

A simulation was performed at the beam current of $180 \mathrm{~mA}$, for different beam sizes. To vary the beam size, the value of the $x y$ coupling was changed which will vary the value of the vertical emittance and thus the vertical beam size. The result for the losses from Touschek scattering as a function of the inverse vertical beam size at $180 \mathrm{~mA}$ is

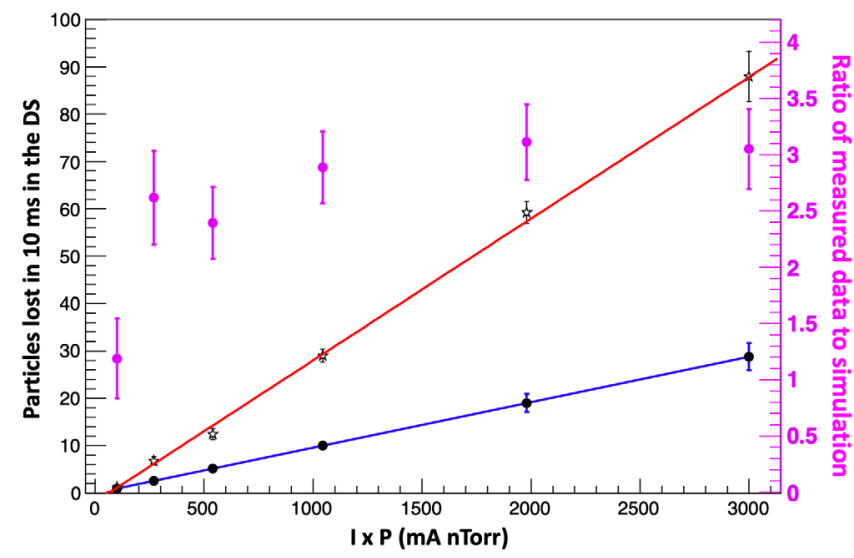

FIG. 18. Losses from beam-gas bremsstrahlung in the SCVD in the LER as a function of the product of the beam current and the vacuum pressure, from measurements in red and from simulations in blue and the ratio of measurement to simulation in magenta.

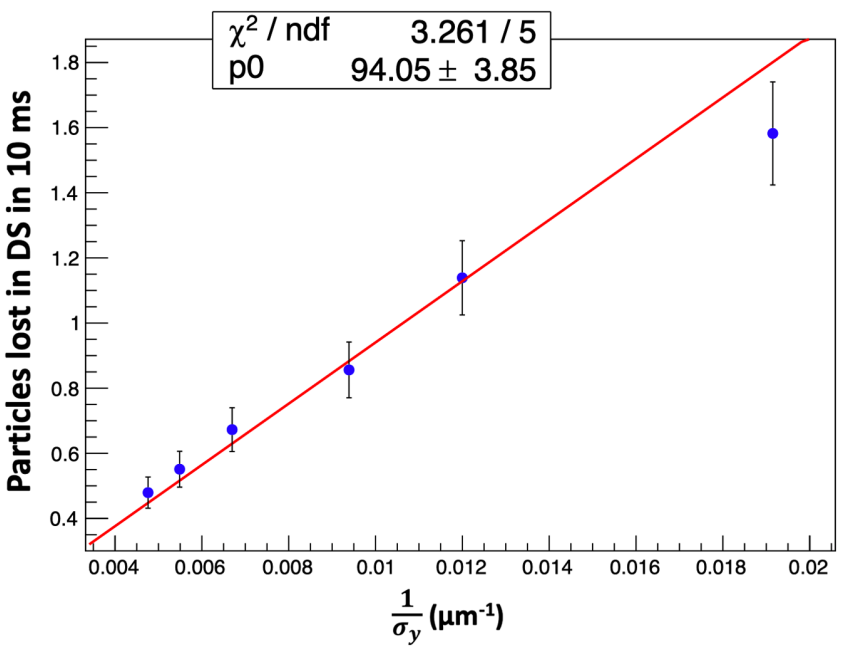

FIG. 19. The simulated losses from Touschek scattering as a function of the inverse vertical beam size at $I=180 \mathrm{~mA}$.

shown in Fig. 19. The result of the fit gives Touschek $_{\text {simu }}=(94.05 \pm 3.85) \times \frac{1}{\sigma_{y}}$. In this scenario, we obtain a factor $\frac{\text { simulation }}{\text { measurement }}=1.33 \pm 0.2$, which shows reasonable compatibility between measurement and simulation within the experimental error, thus confirming the reliability of our simulation tools to extrapolate to the subsequent commissioning phases of SuperKEKB.

\section{CONCLUSION}

Single beam loss measurements were performed successfully during the Phase I commissioning of SuperKEKB. Measurements were done using $500 \mu \mathrm{m}$ diamond sensors placed 11.9 meters downstream of the IP in the LER. Data analysis of Touschek scan studies enabled extracting the loss contributions in our DS from both beam-gas bremsstrahlung and Touschek scattering. Moreover, simulations were reasonably consistent with measurements, within statistical uncertainties. The qualitative and quantitative agreement between our simulations and the measurements enabled us to make preliminary estimates of the level of background in the luminosity monitor diamond sensors that could be expected during the subsequent phases of commissioning and operation of SuperKEKB. The background signal is expected to be two orders of magnitude smaller than the radiative Bhabha scattering signal. This satisfies the conditions specified to achieve a high relative precision on the luminosity measurement.

\section{ACKNOWLEDGMENTS}

We would like to warmly thank K. Kanazawa and Y. Suetsugu for the fruitful discussions and collaboration, and for their encouragements. Part of this work was supported by the P2IO LabEx (ANR-10-LABX-0038) in the framework "Investissements d'Avenir" (ANR11-IDEX-0003-01) managed by the French National 
Research Agency (ANR), by JENNIFER, a MSCA-RISE project of the European Commission, Grant Agreement No. 644294, and by the Toshiko Yuasa France Japan Particle Physics Laboratory (TYL-FJPPL project A_RD_08).

[1] Y. Ohnishi et al., Accelerator design at SuperKEKB, Prog. Theor. Exp. Phys. 2013, 3A011 (2013).

[2] Y. Funakoshi et al., Recent progress of dithering system at SuperKEKB, in Proceedings of IPAC2017, TUPIK059, Copenhagen, Denmark, pp. 1827-1829, http://accelconf .web.cern.ch/AccelConf/ipac2017/papers/tupik059.pdf.

[3] Y. Funakoshi et al., Interaction point orbit feedback system at SuperKEKB, in Proceedings IPAC 2015, MOPHA054, Richmond, Virginia, United State, pp. 921-923, http:// accelconf.web.cern.ch/AccelConf/IPAC2015/papers/mop ha054.pdf.

[4] T. Hirai, S. Uehara, and Y. Watanabe, Real-time luminosity monitor for a B-factory experiment, Nucl. Instrum. Methods Phys. Res., Sect. A 458, 670 (2001).

[5] D. El Khechen, C. Rimbault, P. Bambade, and D. Jehanno, Fast luminosity monitoring using diamond sensors for the super flavour factory SuperKEKB, in Proceedings IPAC14, THPME090, Dresden, Germany, pp. 3442-3444, http://accelconf.web.cern.ch/AccelConf/IPAC2014/papers/ thpme090.pdf.

[6] M. Boscolo, H. Burkhardt, and M. Sullivan, Machine detector interface studies: Layout and synchrotron radiation estimate in the future circular collider interaction region, Phys. Rev. Accel. Beams 20, 011008 (2017).

[7] S. Tanaka, H. Nakayama, Y. Funakoshi, T. Browder, I. Jaegle, S. Vahsen, D. Cinabro, F. H. Lin, M. Z. Wang, and C. Marinas, BEAST II Technical Design Report DRAFT For use in US Belle II Project TDR, 2013.

[8] Beast II Group, First measurements of beam backgrounds at SuperKEKB, Nucl. Instrum. Methods Phys. Res., Sect. A 914, 69 (2019).
[9] Y. Funakoshi et al., Beam commissioning of SuperKEKB, in Proceedings IPAC 2016, TUOBA01, Busan, Korea, pp. 1019-1021, https://accelconf.web.cern.ch/AccelConf/ ipac2016/papers/tuoba01.pdf.

[10] http://acc-physics.kek.jp/SAD/.

[11] D. El Khechen, Ph.D. thesis, Fast luminosity monitoring using diamond sensors for SuperKEKB, Université Paris Saclay, LAL, Orsay, 2016.

[12] M. Friedl, Diploma thesis, Diamond detectors for ionizing radiation, University of Technology, Vienna, 1999.

[13] https://cividec.at/.

[14] M. Pisharody, P. K. Job, S. Magill, J. Proudfoot, and R. Stanek, Measurement of gas bremsstrahlung from electron storage rings, Nucl. Instrum. Methods Phys. Res., Sect. A 401, 442 (1997).

[15] J. Le Duff, Single and multiple Touschek effects, Rhodos 1993, Advanced accelerator physics, Vol. 2*, pp. 573-586, https://cds.cern.ch/record/399406/files/p573.pdf; CERN Geneva Report No. CERN-95-06, 1996, pp. 573-586.

[16] S. C. Leemann, Interplay of Touschek scattering, intrabeam scattering, and $\mathrm{rf}$ cavities in ultralow-emittance storage rings, Phys. Rev. ST Accel. Beams 17, 050705 (2014).

[17] A. Piwinski, J. D. Bjorken, and S. K. Mtingwa, Wilson Prize article: Reflections on our experiences with developing the theory of intrabeam scattering, Phys. Rev. Accel. Beams 21, 114801 (2018).

[18] R. Yang et al., Evaluation of beam halo from beam-gas scattering at the KEK Accelerator Test Facility, Phys. Rev. Accel. Beams 21, 051001 (2018).

[19] Y. Suetsugu (private communication); K.-i. Kanazawa, S. Kato, Y. Suetsugu, H. Hisamatsu, M. Shimamoto, and M. Shrai, Experience of operating the large vacuum system of the KEKB Collider, J-STAGE 45, 149 (2002); K.-i. Kanazawa, Y. Suetsugu, S. Kato, K. Shibata, T. Ishibashi, H. Hisamatsu, M. Shirai, M. Shimamoto, M. Satoh, M. Nishiwaki, and S. Terui, Experiences at the KEK B-factory vacuum system, Prog. Theor. Exp. Phys. 2013, 3A005 (2013). 